\title{
Simulation and On-line Real-time Measurement of Aspirin Crystalline Particle Size Distribution Based on Ultrasound
}

\author{
San $\mathrm{Xu}$ and Xiuxi $\mathrm{Li}^{*}$ \\ School of Chemistry and Chemical Engineering, South China University of Technology, Guangzhou 510640, China \\ ${ }^{*}$ Corresponding author
}

\begin{abstract}
Ultrasound is a new method in the measurement of a crystallization process. The cooling crystallization of Aspirin in ethanol is investigated vias experiments and population balance model. The particle size distribution of the crystallization is monitored online by ultrasonic attenuation spectroscopy. Solubility is measured in situ by the Attenuated Total Reflectance Fourier Transform Infrared. The crystallization kinetics of the Aspirin is studied based on crystallization experiments by a combination of a population balance model and a multiple linear least square method. The results show that the measured value is in good consistent with the simulated value. This proves high feasibility and reliability of ultrasound in the measurement of the crystallization process.
\end{abstract}

Keywords-ultrasound; crystallization; measurement; simulation; particle size distribution

\section{INTRODUCTION}

Crystallization is an important chemical operating unit widely used in the separation and purification of value-added pharmaceuticals, fine chemicals and materials. On-line realtime spectroscopic instrument is a Process Analytical Technology (PAT) [1]. It has been broadly employed in crystallization process optimization and control for preparing high quality crystalline products. Different process analytical technologies have been applied to crystallization processes. For example, the Attenuated Total Reflectance Fourier Transform Infrared (ATR-FTIR) [2-5] and the Ultraviolet-visible spectroscopy (Uvvis) [6-8] were used to measure solution concentration and supersaturation, while the Focused Beam Reflectance Measurement (FBRM) [9-11] was used for the characterization of the size of crystals. And Raman spectroscopy and XRD $[12,13]$ were used for the identification of polymorphs. In recent years, on-line Ultrasonic Attenuation Spectroscopy (UAS)[14-16] has attracted more and more attention for the characterization of crystal size and size distribution. Crystal size distribution is a vital property for crystalline products. Since it can have impact on dissolution rate, processing ability (e.g. filtration efficiency and flowability), and drug bioavailability.

Using ultrasound for particle measurement has many advantages, similar to the light scattering method. Because sound waves can penetrate most of materials, such as colored material or even opaque substances, making ultrasonic measurement as a non-dilution, fast and reliable on-line particle size and Particle Size Distribution (PSD) measurement technology [17]. The purpose of this study is to explore the application of UAS in the crystallization process: kinetics, online real-time measurement and simulation of particle size and PSD. A new developed ultrasound-based device for measuring the PSD of crystallization is used in the crystallization of the Aspirin (ASA) in ethanol. From the microscopic nucleation and growth law, the kinetic model of crystallization process is established based on the experiment and the Population Balance Model (PBM). The finite difference method is used to solve the particle balance equation, thereby simulating the PSD of the actual crystallization process. The feasibility and reliability of ultrasonic application in on-line measurement of crystallization process are verified through comparison of the simulated and experimental values.

\section{UltrasonNIC MEASUREMENT PRINCIPLE}

Its strength will change when the sound waves penetrate the suspension. The attenuation of sound waves can be measured. There are many mathematical models for particle two-phase flow system. The ECAH model is getting increasing attention [18]. Through the processing of a mathematical model related to the propagation of ultrasonic waves in suspension, the ultrasonic propagation velocity and attenuation coefficient are correlated with the particle characterization, such as particle size distribution and concentration [19]. Its measurement principle can be described as: the physical properties of the continuous phase and the particle are known. A suitable theoretical model is then described about the propagation of sound waves in suspension liquid, which has an arbitrary predetermined PSD and concentration. Finally, the predicted theoretical sound attenuation and velocity spectra are obtained. For data reproduction, this step usually gets a model matrix which reflects the relationship between particle system and sound attenuation. The acoustic attenuation coefficient and the velocity of the unknown samples were then measured with the model matrix, the PSD and concentration were obtained by inversion of the data. The above process is illustrated by the following figure. 


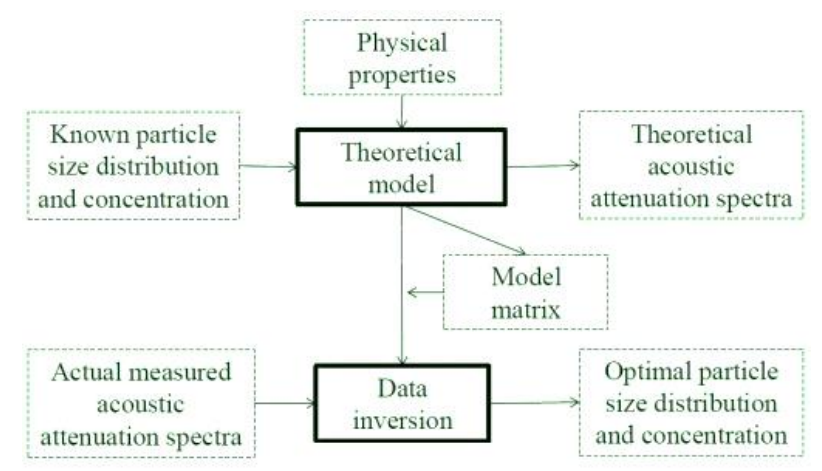

FIGURE I. ULTRASONIC PARTICLE MEASUREMENT PRINCIPLE SHEMATIC

\section{EXPERIMENTAL SECTION}

\section{A. Experimental Device}

The experiment were carried out in a jacketed $1000 \mathrm{~mL}$ glass. The liquid in the glass is stirred with a stirring rate of $0 \sim$ $3500 \mathrm{r} \cdot \mathrm{min}^{-1}$. The crystallization temperature is controlled by the circulating water bath with measuring accuracy of \pm $0.01{ }^{\circ} \mathrm{C}$. The reaction vessel was equipped with an UAS and ATR-FTIR probe. An ATR-FTIR ReactIR 4000 system from Mettler-Toledo (Schwerzenbach, Switzerland) was used. Nanosonic(National Instruments, Pharmavision (QINGDAO) intelligent technology LTD, China) is used for in situ measurements of the PSD. The volume particle size distribution is measured by this equipment using the ECAH model with the range of $20 \mathrm{~nm}-1000 \mu \mathrm{m}$ in high resolution. Meanwhile, the particles in the solution are monitored on-line by the probe-type turbidity meter (Trb 8300, Mettler-Toledo, Switzerland), which is used to determine the nucleation time and calculate the nucleation time.

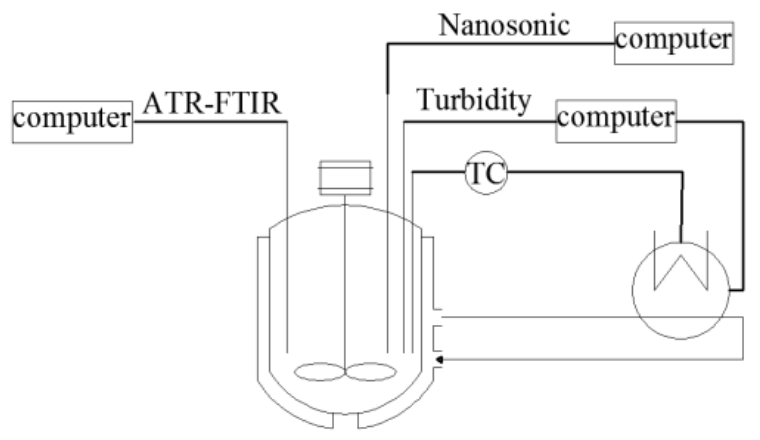

FIGURE II. EXPERIMENTAL SET-UP OF ASPIRIN CRYSTALLIZATION KINETICS.

\section{B. Materials}

Acetylsalicylic acid ( $>99 \%$, Aladdin Industrial Corporation, China), ethanol (99.9\%, Sinopharm Chemical Reagent, China), and deionized water were used in all experiments.

\section{Experimental Program}

A series of cooling crystallization experiments was carried out at different temperatures $\left(25-50{ }^{\circ} \mathrm{C}\right)$ and stirring rates $\left(100-200 \mathrm{r} \cdot \mathrm{min}^{-1}\right)$ to determine kinetics and thermodynamic data. In each of these experiments, a certain amount of Aspirin crystals was dissolved completely in the ethanol by heating it up. The solution was severe-cooled (at a rate of $0.2{ }^{\circ} \mathrm{C} \cdot \mathrm{min}^{-1}$ ) to the desired set-temperature. Then temperature was kept constant. The cooling process was monitored using Nanosonic and ATR-FTIR. At the end of each experiment, the final particle size is extracted and observed by a microscope to measure particle size by off-line sampling of the particle (a certain time interval). All the data were collected to calculate the suspension density.

\section{KinETICS OF CRYSTALLIZATION}

Crystallization kinetics studies the basic law of nucleation and crystal growth, which is the basis of the simulation of crystallization. In any crystallization circumstance, many different phenomena may be scanning, including: nucleation, growth, breakage, agglomeration and dissolution. These phenomena form the kinetic sub models in crystallization. The description of phenomena confirms the description of kinetic formulas applied to the PBM. Nucleation and growth are the two primary phenomena and they are surrounded by masses of crystallization models found in literatures. These rates are contradictive synchronously [20,21], and are identified by detailed functions. Miscellaneous nucleation and growth rate models have been recorded in literatures [22, 23]. According to the description of material being crystallized, the parameters of these models are different. In this work, the ASA-ethanol system will be the system used. In this study, the authors use the commonly empirical models ((1) and (2)) for both kinetic rates.

$$
B=k_{b} M_{T}^{i} N_{T}^{j}\left(c-c^{*}\right)^{b}
$$

$$
G=k_{g}\left(c-c^{*}\right)^{g}
$$

Where $c$ is the solute concentration in the solution and $c^{*}$ is the saturation concentration. It must be noted that the effects of the suspension density, $M_{T}$ and the mixing rates, $N_{T}$ on the nucleation rate are included in (1). $K_{b}, b, k_{g}$ and $g$ are unknown kinetic parameters to be determined from experiments.

\section{A. Population Balance Model}

Seeing the crystallization process is a particulate one, Hulbert and Katz [24] devised the population balance theory as a method to modeling of the crystallization process. This theory suggested the method to use a balance to confirm the number of crystals. According to the balance, a distribution of crystals in two scales will be obtained. This theory can generate the conclusive presentation of the totally dynamic distribution of particle size.

According to the book named Theory of Particulate Processes, Randolph and Larson [25] explain the development of the population balance model. For batch cooling 
crystallization with size independent growth, the resulting crystal population balance equation (PBE) is shown as below.

$$
\frac{\partial n(L, t)}{\partial t}=-G \frac{\partial n(L, t)}{\partial L}+B
$$

The first term in (3) represents the accumulation of crystal numbers in the size range from $L_{i-1}$ to $L_{i}$, while the second term represents the net flux of particles through the size range with crystal growth $(G)$. The third term, $B$ represents birth of crystals.

\section{B. Moment Method}

The moment method reported by Hulbert and Katz [24] and later modified by others [25] can frequently be applicable to calculate the nucleation rate $B$ and growth rate $G$ from the PSD. The $j$ th moment is defined as

$$
m_{j}=\int_{0}^{\infty} n(L, t) L^{j} d L
$$

Where the first four moments are of extraordinary interest; they have relation to total number, length, area, and volume of solid per unit volume of suspension. Randolph and Larson [25] showed that for nucleation and growth alone, the moments may be produced by the ordinary differential equation.

$$
d m_{j} / d t=0^{j} B+j G m_{j-1} .
$$

When the time interval is small, (5) can be rewritten as:

$$
\begin{gathered}
\Delta m_{0} / \Delta t=B . \\
\Delta m_{3} /\left(3 m_{2} \Delta t\right)=G .
\end{gathered}
$$

\section{Kinetics Data Processing}

\section{A. Solubility and Supersolubility}

In this study, the gravimetry was applied to measure the solubility of ASA in the ethanol within the temperature range from 298.15 to $339.15 \mathrm{~K}$. The measurement of the supersolubility of ASA in ethanol is based on turbidity meter. Measurement of supersolubility can determine the width of metastable zone. The key of the former is to capture the visual image of the first batch of nuclei, and the measurement of supersolubility will influence the stirring rate and experimental temperature. The measured metastable zone is shown in Figure 3. From this figure, the solubility of ASA in ethanol solution increases as the increase of temperature. The solubility equation is as follows:

$$
x=2789.5936-20.4857 T+0.0380 T^{2}\left(R^{2}=0.998\right) .
$$

Where $x$ is the mole fraction of ASA in solution, $\mathrm{T}$ is the solution equilibrium temperature.

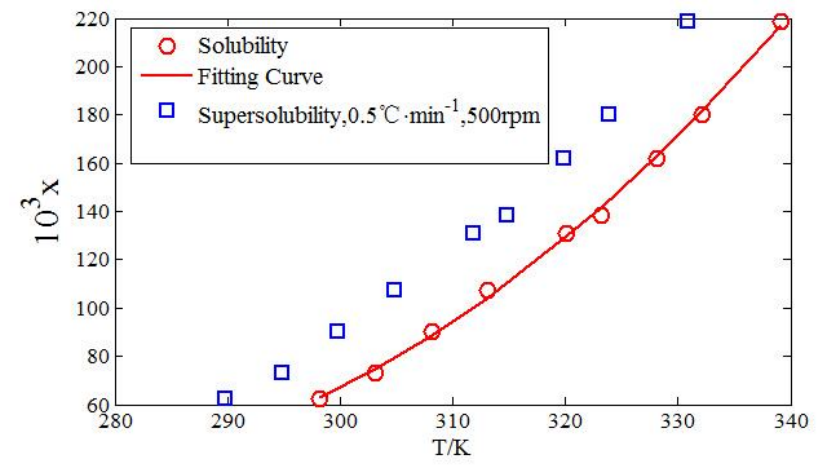

FIGURE III. MEASURED SOLUBILITY AND SUPERSOLUBILITY OF ASA IN ETHANOL.

\section{B. Particle Number Density}

The particle size distribution was measured by using UAS in situ. The output result of this device is the volume PSD. (9) can transform the volume of PSD to the number of PSD, we use the number of PSD in this paper:

$$
n_{i}=\frac{M_{T} V_{i}}{\rho_{c} k_{V} L_{i}^{3} \Delta L_{i}} .
$$

Where $V_{i}$ is the volume fraction of the particle in the $i$ th size range, $L_{i}$ is the arithmetic mean of the upper and lower sizes of the $i$ th size range and $\Delta L_{i}$ is the difference between them

\section{Slurry Suspension Density}

The suspension density is the sum of the crystal quality in the whole particle size range per unit volume. It is an important parameter for nucleation (see (1)), and a very important operational parameter for the crystallizer. The suspension density can be calculated by the following formula:

$$
M_{T}=k_{V} \rho_{c} \int_{0}^{\infty} n L^{3} d L
$$

\section{Parameter Estimation}

For a series of kinetic data obtained under different experimental conditions, such as the solution supersaturation $\triangle C$, the suspension density $M_{T}$. The equation of $G$ and $B$ are shown in (6) and (7) with the results of particle size analysis, the kinetic parameters of empirical formula (1) and (2) were obtained by multiple linear regression analysis.

$$
G=9.695 \times 10^{-9} \Delta C^{1.56}\left(R^{2}=0.946\right) .
$$

$$
B=1.23 \times 10^{8} M_{T}^{0.97} N_{T}^{2.5} \Delta C^{1.78}\left(R^{2}=0.831\right) .
$$




\section{E. Comparison of Experimental Result and Simulation}

In order to further illustrate the reliability and feasibility of the new crystallization kinetic measurement device with ultrasound in this study, the experimental and simulated values of the growth rate and nucleation rate were shown in Figures 4 and 5. As can be seen from these figures, the points (open and filled circles) are basically close to the diagonal. This means that calculation results are consistent with the experiment. The relative errors are drawn in the figure by the dotted line. Due to the randomness and complexity of the crystallization process, the relative errors in the initial stage of crystallization are larger, and then it is in a small range.

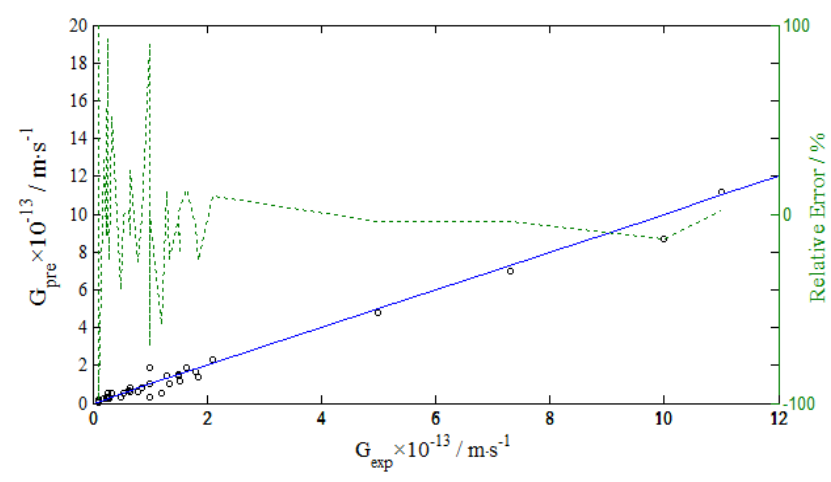

FIGURE IV. EXPERIMENTAL GROWTH RATES VERSUS PREDICTIVE VALUE FROM THE KINETIC MODEL

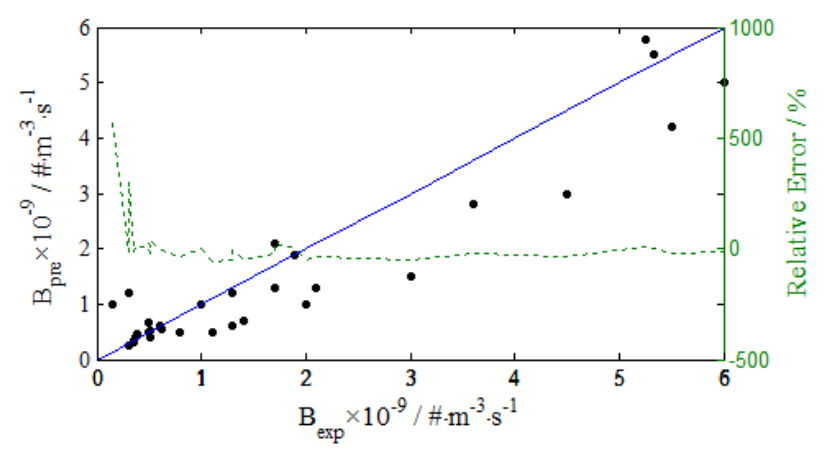

FIGURE V. EXPERIMENTAL NUCLEATION RATES VERSUS PREDICTIVE VALUE FROM THE KINETIC MODEL

\section{Simulation of the Particle Size Distribution}

\section{A. Discretisation of the Population Balance}

According to Ali and Jose [26], the PBE is transformed into a set of linear differential equations by means of discretisation. As can be seen from below, the distance between discrete crystal sizes is determined by a geometric series. It is very important that this family of equations can be used to design (and modify) the range of any crystal size distribution. The discrete form of (3) and the general equation describing the size of a crystal is:

$$
\frac{d N_{1}}{d t}=-\frac{G}{2 \phi_{1}} N_{1}+B
$$

$$
\begin{gathered}
\frac{d N_{i}}{d t}=-\frac{G}{2 \phi_{i}} N_{i}+\frac{G}{2 \phi_{i-1}} N_{i-1}, i=2,3, \ldots, m-1 . \\
\frac{d N_{m}}{d t}=-\frac{G}{2 \phi_{m}} N_{m}+\frac{G}{2 \phi_{m-1}} N_{m-1} . \\
\phi_{i}=L_{i}-L_{i-1} .
\end{gathered}
$$

$$
L_{i}=a b^{i}, i=0,1,2, \ldots, m
$$

$$
L_{0}=a
$$

Where $N_{i}$ represents the total number of particles in the $i$ th size interval from $L_{i-1}$ to $L_{i}, L_{i}$ is the size (m) of the $i$ th size range. $L_{0}$ is the lowest size at which the measurement device registers a reading.

The solute concentration in ethanol, $c$, can be determined by the material balance by the following equation:

$$
d c / d t=-\frac{3}{M} k_{V} \rho_{c} G \int_{0}^{\infty} n L^{2} d L
$$

Where $M$ is molecular mass of ASA, $k_{V}$ is the volume shape factor, and $\rho_{c}$ is the crystal density. In this work we set the crystal density $\rho_{c}$ equal to $1350 \mathrm{~g} \cdot \mathrm{kg}^{-1}$ and the volume shape factor $k_{V}$ to $\pi / 6$. The following initial and boundary conditions are used to the PBE and the material balance:

$$
\begin{gathered}
n\left(L_{0}, t\right)=B / G . \\
n(L, 0)=n_{0} .
\end{gathered}
$$

$$
c(0)=c_{0}
$$

Where $B$ is the nucleation rate, and $n_{0}$ and $c_{0}$ are the initial PSD and solute concentration.

\section{B. Measurement and Simulation Results of PSD}

PSDs of the crystallization at different times are measured and results are shown in Figure 6. At the early stage (the first $10 \mathrm{~min})$, the solution is burst into nuclei because of the high degree of saturation, and the emergence of a large number of nuclei makes the size range of the particle distribution smaller. The formation of crystal nucleus causes the decrease of saturation of the solution. As time elapses, the system enters a stable period of crystal nucleation and growing. At $90 \mathrm{~min}$, the PSD is concentrated, and the size range of the particle distribution is larger. 


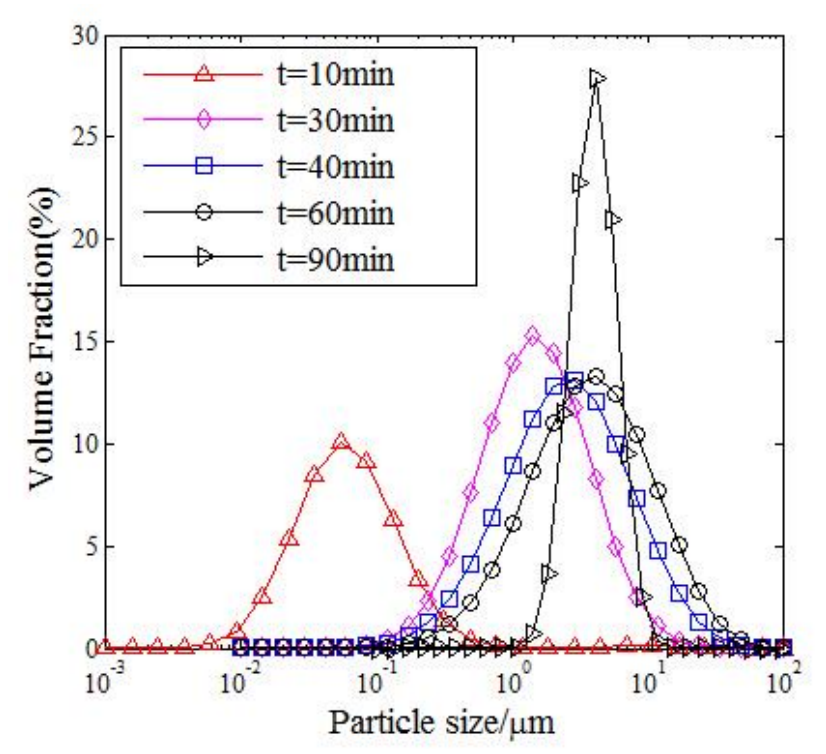

FIGURE VI. EVOLUTION OF THE PSD BASED ON EXPERIMENTS

Combined with the research system and the kinetics experimental results, we set $L_{0}$ equal to $0.06 \mu \mathrm{m}$ for the nucleus size and $L_{m}$ equal to $1000 \mu \mathrm{m}$ for the largest crystal size. Here, $m$ is equal to 300 for the finite element numbers. The geometry constant $\mathrm{b}$ can be calculated by (17):

$$
b=150 \sqrt{\frac{1000}{0.02}}=1.0748 .
$$

Equations (13) - (18) can be solved together with (19) using MATLAB ode45 solver. We compared the PSD measured by the UAS and the simulated result obtained by the finite difference method (FD). The results is shown in Figure 7 for a cooling rate of $0.2{ }^{\circ} \mathrm{C} \cdot \mathrm{min}^{-1}$ and a stirring rate of 100 $\mathrm{r} \cdot \mathrm{min}^{-1}$. There is a good agreement between the two sets of data.

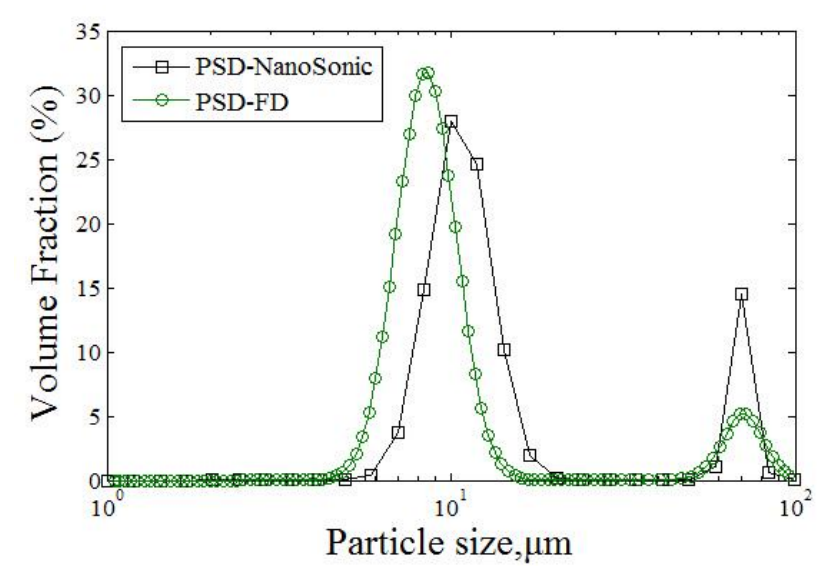

FIGURE VII. COMPARISION OF THE PSD MEASURED BASED ON THE UAS AND MODEL

The change of PSD in different time periods is then shown in Figure 8 (The small chart at the upper right corner is magnified by the final state of PSD). The width where the volume fraction of the PSD is 5\% is marked at the 90s min and the end time, respectively. The former is $6.4 \mu \mathrm{m}$, the latter is about $5.3 \mu \mathrm{m}$. PSD is getting narrower. At the $10 \mathrm{~s} \mathrm{~min}$, the early growth (nucleation dominated), the bulk of the crystal nucleus consume the solute rapidly, and the supersaturation changes obviously. So that the particle is concentrated in the small size region, the PSD curve is wide, and the distribution is scattering. At the end of the crystallization, as $t$ equal to $140 \mathrm{~s}$ min, the crystal sizes are uniform, when the distribution is concentrated. A comparison of the simulated and experimental (see Figure 6) PSD profiles for different times shows that the former one has a good consistent with the crystallization mechanism. This illustrates that there is a feasibility and reliability in real-time on-line measurement of the PSD in the crystallization based on ultrasound. As can be seen on the microscope pictures in Figure 9, the mean size of the final crystals is $85 \mu \mathrm{m}$. A good agreement is observed with Figure 8 .

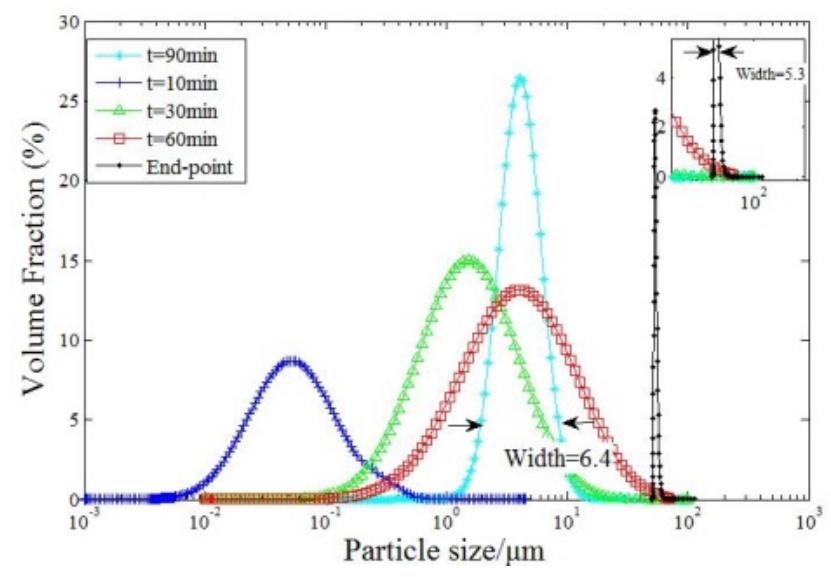

FIGURE VIII. EVOLUTION OF THE PSD BASED ON SIMULATION

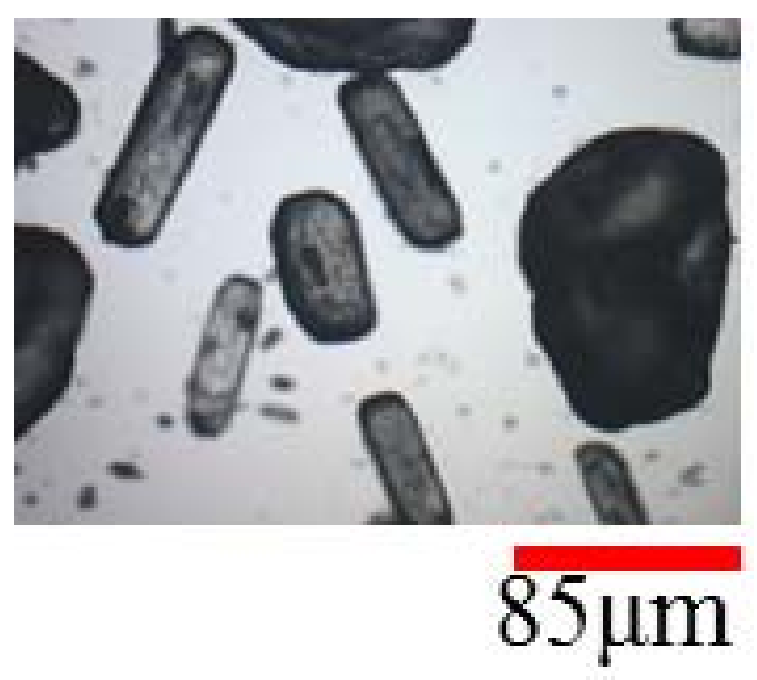

FIGURE IX. MICROSCOPE PICTURES OF THE FINAL ASA CRYSTALS

\section{CONCLUSIONS}

This work analysed the reliability and feasibility of ultrasound as a new on-line measurement method used in the 
measurement and simulation of the PSD in the crystallization. The batch cooling crystallization of ASA in ethanol was monitored by two in situ process analytical technologies, ATRFTIR spectroscopy and Nanosonic which is based on UAS. In terms of thermodynamics, the solution metastable zone was determined by measuring the solubility and supersolubility. As for kinetics, the nucleation rate and growth rate equation were obtained vias multiple linear regression analysis.

A comparision of experimental and simulated result for the nucleation rate and the growth rate is conducted. The relative error was small, showing a good agreement of the model. Finally, the PSD of ASA at different times at a certain crystallization condition was calculated by the PBM, and compared with the experimental results. It also showed a good agreement.

\section{TABLE I. ABBREVIATIONS AND ACRONYMS}

$\begin{array}{ll}A S A & \text { aspirin } \\ B & \text { nucleation rate }\left(\# \cdot \mathrm{m}^{-3} \cdot \mathrm{s}^{-1}\right) \\ G & \text { growth rate }\left(\mathrm{m} \cdot \mathrm{s}^{-1}\right) \\ k_{V} & \text { volume shape factor }(-) \\ L_{i} & \text { size of the } i \text { th size range }(\mathrm{m}) \\ M_{T} & \text { suspension density }\left(\mathrm{g} \cdot \mathrm{kg}^{-3}\right) \\ m & \text { finite elements number }(\#) \\ N_{T} & \text { stirring rate }\left(\mathrm{rpm} \cdot \mathrm{min}^{-1}\right) \\ N_{i} & \text { number of particles of the } i \text { th size range }(\#) \\ n_{i} & \text { ith moment of the } \mathrm{PSD}\left(\# \cdot \mathrm{m}^{-3} \cdot \mathrm{m}^{-1}\right) \\ P B E & \text { population balance equation } \\ P B M & \text { population balance model } \\ P S D & \text { particle size distribution } \\ T & \text { temperature }(\mathrm{K}) \\ t & \text { time }(\mathrm{s}) \\ U A S & \text { Ultrasonic attenuation spectroscopy } \\ V_{i} & \text { volume fraction of particles in the } i \text { th size range } \\ \rho_{c} & \text { ( \% ) } \\ & \text { crystal density }\left(\mathrm{g} \cdot \mathrm{kg} \mathrm{g}^{-3}\right)\end{array}$

\section{ACKNOWLEDGMENT}

This work was financially supported by the China NSF projects (21376091) and the Natural Science Foundation Team Project of Guangdong Province (S2011030001366).

\section{REFERENCES}

[1] Food and D. Administration, "Guidance for industry: PAT-A framework for innovative pharmaceutical development, manufacturing, and quality assurance," DHHS, Rockville, MD, 2004.

[2] D.-T. Tan, C. Cai, Y. Zhang, N. Wang, S.-F. Pang, and Y.-H. Zhang, "Crystallization kinetics from mixture Na2SO4/glycerol droplets of Na2SO4 by FTIR-ATR," Chemical Physics, vol. 475, pp. 131-135, Aug 222016
[3] T. B. Hansen, A. Taris, B.-G. Rong, M. Grosso, and H. Qu, "Polymorphic behavior of isonicotinamide in cooling crystallization from various solvents," Journal of Crystal Growth, vol. 450, pp. 81-90, Sep 152016.

[4] D. J. Griffin, M. A. Grover, Y. Kawajiri, and R. W. Rousseau, "Masscount plots for crystal size control," Chemical Engineering Science, vol. 137, pp. 338-351, Dec 12015.

[5] C. Moriwaki, C. S. Mangolim, G. B. Ruiz, G. R. de Morais, M. L. Baesso, and G. Matioli, "Biosynthesis of CGTase by immobilized alkalophilic bacilli and crystallization of beta-cyclodextrin: effective techniques to investigate cell immobilization and the production of cyclodextrins," Biochemical Engineering Journal, vol. 83, pp. 22-32, 2014.

[6] E. Simone, M. V. Cenzato, and Z. K. Nagy, "A study on the effect of the polymeric additive HPMC on morphology and polymorphism of orthoaminobenzoic acid crystals," Journal of Crystal Growth, vol. 446, pp. 50-59, Jul 152016.

[7] E. Mielniczek-Brzoska, "Effect of sample volume on the metastable zone width of potassium nitrate aqueous solutions," Journal of Crystal Growth, vol. 401, pp. 271-274, Sep 12014.

[8] B. L. Shivachev, K. Kossev, L. T. Dimowa, G. Yankov, T. Petrov, R. P. Nikolova, et al., "Synthesis, growth, structural, thermal, optical properties of new metal-organic crystals: Methyltriphenylphosphonium iodide thiourea and methyltriphenylphosphonium iodide chloroform hemisolvate," Journal of Crystal Growth, vol. 376, pp. 41-46, Aug 1 2013.

[9] Y. Zhang, Y. Jiang, D. Zhang, Y. Qian, and X. Z. Wang, "Metastable zone width, crystal nucleation and growth kinetics measurement in antisolvent crystallization of $\beta$-artemether in the mixture of ethanol and water," Chemical Engineering Research and Design, vol. 95, pp. 187194, 2015.

[10] O. S. Agimelen, P. Hamilton, I. Haley, A. Nordon, M. Vasile, J. Sefcik, et al., "Estimation of particle size distribution and aspect ratio of nonspherical particles from chord length distribution," Chemical Engineering Science, vol. 123, pp. 629-640, Feb 172015.

[11] C. Lindenberg, M. Kraettli, J. Cornel, M. Mazzotti, and J. Brozio, "Design and Optimization of a Combined Cooling/Antisolvent Crystallization Process," Crystal Growth \& Design, vol. 9, pp. 11241136, Feb 2009.

[12] D. Zhang, T. Liu, S. Chen, M. Miao, J. Cheng, A. Zhang, et al., "Aminoended hyperbranched polyamide as template for tuning the morphology of self-assembled ZnS particles," Materials Chemistry and Physics, vol. 184, pp. 162-171, Dec 12016.

[13] Ratnawati, J. Gunlazuardi, and Slamet, "Development of titania nanotube arrays: The roles of water content and annealing atmosphere," Materials Chemistry and Physics, vol. 160, pp. 111-118, Jun 152015.

[14] X. Z. Wang, L. Liu, R. F. Li, R. J. Tweedie, K. Primrose, J. Corbett, et al., "Online characterisation of nanoparticle suspensions using dynamic light scattering, ultrasound spectroscopy and process tomography," Chemical Engineering Research \& Design, vol. 87, pp. 874-884, Jun 2009.

[15] P. Mougin, D. Wilkinson, and K. J. Roberts, "In situ measurement of particle size during the crystallization of L-glutamic acid under two polymorphic forms: Influence of crystal habit on ultrasonic attenuation measurements," Crystal Growth \& Design, vol. 2, pp. 227-234, May-Jun 2002.

[16] P. Hauptmann, N. Hoppe, and A. Püttmer, "Application of ultrasonic sensors in the process industry," Measurement Science and Technology, vol. 13, p. R73, 2002.

[17] B. Boonkhao, "On-line characterisation techniques for manufacture of nanomaterials," University of Leeds, 2011.

[18] A. H. Harker and J. A. G. Temple, "Velocity and attenuation of ultrasound in suspensions of particles in fluids," Journal of Physics D (Applied Physics), vol. 21, pp. 1576-88, 14 Nov. 1988.

[19] J. S. Tebbutt and R. E. Challis, "Ultrasonic wave propagation in colloidal suspensions and emulsions: a comparison of four models," Ultrasonics, vol. 34, pp. 363-8, June 1996. 
[20] T. Kilpio and H. V. Nordén, "Simulation and control of continuous crystallisation," ACTA POLYTECHNICA SCANDINAVICACHEMICAL TECHNOLOGY SERIES, pp. 1-49, 1996.

[21] J. Nývlt, Industrial crystallisation: the state of the art: Wiley-VCH, 1982.

[22] M. Aoun, E. Plasari, R. David, and J. Villermaux, "A simultaneous determination of nucleation and growth rates from batch spontaneous precipitation," Chemical Engineering Science, vol. 54, pp. 1161-1180, May 1999.

[23] S. T. Liu, G. H. Nancollas, and E. A. Gasiecki, "Scanning electron microscopic and kinetic studies of the crystallization and dissolution of barium sulfate crystals," Journal of Crystal Growth, vol. 33, pp. 11-20, April 1976.

[24] H. M. Hulburt and S. Katz, "Some problems in particle technology: A statistical mechanical formulation," Chemical Engineering Science, vol. 19, pp. 555-574, 8// 1964.

[25] A. Ranodolph, Theory of Particulate Processes 2e: Analysis and Techniques of Continuous Crystallization: Elsevier, 2012.

[26] A. Abbas and J. A. Romagnoli, "Multiscale modeling, simulation and validation of batch cooling crystallization," Separation and Purification Technology, vol. 53, pp. 153-163, Feb 252007. 